\title{
BMJ Open Spatial clustering of drug-resistant tuberculosis in Hunan province, China: an ecological study
}

\author{
Kefyalew Addis Alene (D) , ${ }^{1,2}$ Zuhui Xu, ${ }^{3}$ Liqiong Bai, ${ }^{4}$ Hengzhong Yi, ${ }^{5}$ \\ Yunhong Tan, ${ }^{5}$ Darren Gray, ${ }^{6}$ Kerri Viney, ${ }^{6,7,8}$ Archie CA Clements ${ }^{1,2}$
}

To cite: Alene KA, Xu Z, Bai L, et al. Spatial clustering of drug-resistant tuberculosis in Hunan province, China: an ecological study. BMJ Open 2021;11:e043685. doi:10.1136/ bmjopen-2020-043685

- Prepublication history and additional material for this paper is available online. To view these files, please visit the journal online (http://dx.doi.org/10. 1136/bmjopen-2020-043685).

Received 13 August 2020 Revised 19 February 2021 Accepted 24 February 2021

\section{Check for updates}

(c) Author(s) (or their employer(s)) 2021. Re-use permitted under CC BY-NC. No commercial re-use. See rights and permissions. Published by BMJ.

For numbered affiliations see end of article.

\section{Correspondence to} Dr Kefyalew Addis Alene; kefyalew.alene@telethonkids. org.au

\section{ABSTRACT}

Objective This study aimed to investigate the spatial distribution of drug-resistant tuberculosis (DR-TB) in Hunan province, China.

Methods An ecological study was conducted using DRTB data collected from the Tuberculosis Control Institute of Hunan Province between 2012 and 2018. Spatial clustering of DR-TB was explored using the Getis-Ord statistic. A Poisson regression model was fitted with a conditional autoregressive prior structure, and with posterior parameters estimated using a Bayesian Markov chain Monte Carlo simulation, to quantify associations with possible risk factors and identify clusters of high DR-TB risk.

Results A total of 2649 DR-TB patients were reported to Hunan TB Control Institute between 2012 and 2018. The majority of the patients were male $(74.8 \%, \mathrm{n}=1983)$ and had a history of TB treatment $(88.53 \%, \mathrm{n}=2345)$. The proportion of extensively DR-TB among all DR-TB was $3.3 \%(95 \% \mathrm{Cl} 2.7 \%$ to $4.1 \%)$, which increased from $2.8 \%$ in 2012 to $4.4 \%$ in 2018. Of 1287 DR-TB patients with registered treatment outcomes, $434(33.8 \%)$ were cured, 198 (15.3\%) completed treatment, 92 (7.1\%) died, $108(8.3 \%)$ had treatment failure and $455(35.3 \%)$ were lost to follow-up. Half $(50.9 \%, \mathrm{n}=655)$ had poor treatment outcomes. The annual cumulative incidence rate of notified DR-TB increased over time from 0.25 per 100000 people in 2012 to 0.83 per 100000 people in 2018. Substantial spatial heterogeneity was observed, and hotspots were detected in counties located in the North and East parts of Hunan province. The cumulative incidence of notified DRTB was significantly associated with urban communities. Conclusion The annual incidence of notified DR-TB increased over time in Hunan province. Spatial clustering of DR-TB was detected and significantly associated with urbanisation. This finding suggests that targeting interventions to the highest risk areas and population groups would be effective in reducing the burden and ongoing transmission of DR-TB.

\section{INTRODUCTION}

Tuberculosis (TB) remains a significant global public health problem that kills more than one million people every year and over two billion people during the last two centuries. ${ }^{12}$ In 2018, one-third of the world's population were estimated to have

\section{Strengths and limitations of this study}

To the best of our knowledge, this is the first study to explore the spatial clustering of drug-resistant tuberculosis (DR-TB) in Hunan province, China.

- Using 6-year surveillance data, this study produced maps showing the counties where DR-TB were clustered, important information to design targeted interventions for the prevention and control of TB and DR-TB.

- The association between potential risk factors and spatial clustering of DR-TB were quantified using model-based geostatistics with a Bayesian Markov chain Monte Carlo simulation.

- The ecological study design did not allow us to determine causation or directionality of association between DR-TB and county-level characteristics.

latent TB infection, and approximately 10 million people developed TB disease. ${ }^{1}$ The United Nations (UN) Sustainable Development Goals and the WHO's End TB Strategy have a common target of ending the global TB epidemic by $2030 .{ }^{34}$ However, the emergence of multidrug-resistant tuberculosis (MDR-TB), defined as TB that is resistant to at least isoniazid and rifampicin, has become a major obstacle to achieve these ambitious targets. Globally, approximately $3.4 \%$ of new TB cases and $18 \%$ of previously treated cases have MDR-TB or rifampicin-resistant TB $(\mathrm{MDR} / \mathrm{RR}-\mathrm{TB}){ }^{1}$ About half a million new cases of MDR/RR-TB were estimated to occur in $2018 .^{1}$

China has the second-largest global burden of DR-TB (after India), accounting for $14 \%$ of the global TB burden. ${ }^{1}$ The country is striving to reduce the magnitude of this burden in line with the targets of the global End-TB Strategy and the national TB strategic plan. ${ }^{4}$ DR-TB in China is unevenly distributed by geographic area and population subgroup, with a higher incidence reported among males, minority ethnic groups, those living in poverty and people with chronic medical 
conditions such as HIV and diabetes mellitus. ${ }^{56}$ The identification of potential risk factors and hotspot areas where TB is concentrated should allow policy makers to implement targeted interventions aimed at DR-TB prevention and management. This might be particularly important in high DR-TB burden countries such as China as the epidemic is concentrated in certain areas. However, the spatial distribution of DR-TB has not been studied in some provinces of China, including in Hunan province.

Hunan is one of the provinces in China with a high burden of DR-TB. ${ }^{7}$ The proportions of DR-TB among all patients with $\mathrm{TB}$ and previously treated patients with TB in Hunan were $10.5 \%$ and $28.8 \%$, respectively. ${ }^{8}$ Our previous studies showed that successful treatment outcomes for DR-TB patients in Hunan Province are low at $57 \%$, suggesting that additional efforts are needed to control and prevent the disease. ${ }^{9} 10$ To inform these efforts, the distribution of DR-TB in terms of place and time as well as the drivers of transmission should be investigated and documented. This study explored the spatial distribution of DR-TB and identified the areas where DR-TB is concentrated in Hunan province. The study aims to provide additional evidence for policy-makers that can help develop targeted interventions to reduce the burden of DR-TB in the province.

\section{METHODS}

\section{Study area}

This study was conducted in Hunan province, 1 of the 22 provinces in China, located in South Central China along the middle reaches of the Yangtze River. Hunan is the seventh largest province in China with a total population of approximately 72 million people and with a total area of 211800 square kilometres. ${ }^{11}$ Hunan province is administratively divided into fourteen prefecture-level divisions (13 prefecture-level cities and one autonomous prefecture); and further subdivided into 122 county-level divisions (35 districts, 17 county-level cities, 63 counties, 7 autonomous counties). Those are in turn divided into 2587 township-level divisions (1098 towns, 1158 townships, 98 ethnic townships, 225 subdistricts and 8 district public offices).

\section{DR-TB diagnosis and treatment in Hunan province}

DR-TB diagnosis and treatment regimen in Hunan Province has been described detailly elsewhere. ${ }^{9}$ Briefly, of the 131 counties in Hunan province, 32 counties are able to provide comprehensive diagnostic services, which include culture. However, drug susceptibility testing (DST) is mainly carried out in the Hunan Chest Hospital. Thus, sputum specimens from all culture-positive TB patients from throughout the province are referred to the Hunan Chest Hospital for DST. In the hospital, phenotypic DST based on solid and liquid culture techniques, and molecular methods using line probe assays as well as Xpert MTB/RIF are performed. Patients with confirmed DR-TB are admitted to the MDR-TB treatment centre at the Hunan Chest Hospital for treatment and management. Patients were treated with an individualised treatment regimen based on their DST results and history of previous TB treatment. The regimen at the time of this study includes an injectable agent, a fluoroquinolone, para-aminosalicylic acid, prothionamide, pyrazinamide, clarithromycin, ethambutol or cycloserine. The duration of treatment was 24 months for patients with MDR-TB and 30 months for patients with extensively DR-TB. The injectable drugs were used for a minimum of 6 months for MDR-TB patients and 12 months for XDR-TB patients. Patients were admitted to the hospital for 1-2 months during the intensive phase. During this time, patients received psychological support and counselling from hospital nurses. When the patients are medically fit, they were treated as outpatients. They received support from trained family members or from trained supervisors in the community and returned to the hospital once a month for a drug refill until the end of treatment.

\section{Study design and data sources}

The study was conducted at the county level, using an ecological study design. DR-TB data were obtained from the internet-based TB management information system managed by the Hunan Tuberculosis Control Institute. The study included information from patients with RR-TB, MDR-TB and XDR-TB that were reported to the Hunan Tuberculosis Control Institute, during the period 2012-2018, as part of routine TB reporting. Population estimates were obtained from the Hunan Bureau of Statistics. A wide range of socioeconomic, environmental, and healthcare access data were also obtained from Hunan Bureau of Statistics website to be used as possible covariates. The TB data obtained from the Hunan Tuberculosis Control Institute were aggregated at the county level and were linked with the possible covariates using ArcGIS Geographical Information System software (ESRI, Redlands, CA). ${ }^{12}$

\section{Data analysis}

The overall crude incidence rate of notified DR-TB was calculated by taking the total number of DR-TB cases reported during the study period as the numerator and the mid-point total population during the same time period as the denominator. The standardised morbidity ratio (SMR) was then calculated for each county using the formula: $\mathrm{Y}_{\mathrm{i}}=\left[\mathrm{O}_{\mathrm{i}} / \mathrm{E}_{\mathrm{i}}\right]$; where $\mathrm{Y}_{\mathrm{i}}$ is the SMR in the county, $\mathrm{O}$ is the observed number of DR-TB cases in the county and $\mathrm{E}$ is the expected number of DR-TB cases in the county across the study period. The expected number of DR-TB cases for each county was calculated by multiplying the mid-point population of each county by the overall crude DR-TB incidence rate for the study area and period.

\section{Spatial analysis}

Spatial autocorrelation was explored using Moran's I statistic and the Getis-Ord statistic. The Moran's I statistic was used to assess the presence, strength and 
Table 1 Sociodemographic and clinical characteristics of patients with drug-resistant tuberculosis in Hunan province, 2012-2018

\begin{tabular}{|cc}
\hline Variables & No (\%) \\
\hline Sex & $1983(74.86)$ \\
\hline Male & $666(25.14)$ \\
\hline Female & $46.54($ SD +15.16$)$ \\
\hline Mean age (years) & \\
\hline Management organisation & $2263(88.09)$ \\
\hline CDC & $306(11.91)$ \\
\hline TB hospital & \\
\hline Notification year & $177(6.68)$ \\
\hline 2012 & $221(8.34)$ \\
\hline 2013 & $352(13.29)$ \\
\hline 2014 & $375(14.16)$ \\
\hline 2015 & $511(19.29)$ \\
\hline 2016 & $406(15.33)$ \\
\hline 2017 & $607(22.91)$ \\
\hline 2018 &
\end{tabular}

Month of TB registration

\begin{tabular}{lr}
\hline January-April & $506(19.10)$ \\
\hline May-August & $914(34.50)$ \\
\hline September-December & $1229(46.40)$ \\
\hline Occupation & \\
\hline Farmer & $1883(71.08)$ \\
\hline Government employee & $76(2.87)$ \\
\hline Non-government employee & $150(5.66)$ \\
\hline Labourer & $74(2.79)$ \\
\hline Retired & $108(4.08)$ \\
\hline Student & $65(2.45)$ \\
\hline Unknown & $113(4.27)$ \\
\hline Others & $180(6.8)$ \\
\hline Type of DR-TB & $121(4.57)$ \\
\hline RR-TB & $2439(92.07)$ \\
\hline MDR-TB & $89(3.36)$ \\
\hline XDR-TB & $1111(41.94)$ \\
\hline History of TB treatment & $1371(51.76)$ \\
\hline First-line treatment history & $974(36.77)$ \\
\hline Second-line treatment history & $304(11.48)$ \\
\hline No TB treatment history & \\
\hline Mode of treatment & \\
\hline Ambulatory & \\
\hline Nospitalisation & \\
\hline China & \\
\hline
\end{tabular}

Continued

\begin{tabular}{lc}
\hline Table 1 Continued & \\
\hline Variables & No (\%) \\
\hline Both & $178(6.72)$ \\
\hline Not registered & \\
Treatment outcomes & $198(7.47)$ \\
Completed treatment & $434(16.38)$ \\
Cure & $108(4.08)$ \\
Failure & $455(17.18)$ \\
Lost to follow-up & $92(3.47)$ \\
death & $25(0.94)$ \\
Unknown & $1337(50)$ \\
Still on treatment
\end{tabular}

.CDC, The Centres for Disease Control and Prevention; DR-TB, drug-resistant tuberculosis; GLC, green light committee; MDRTB, multidrug-resistant tuberculosis; RR-TB, rifampicin resistant tuberculosis; TB, tuberculosis; XDR-TB, extensively drug-resistant tuberculosis.

direction of spatial autocorrelation over the whole study area and to test the assumption of spatial independence in the implementation of the spatial pattern analysis. The spatial clustering analysis using Getis-Ord Gi* statistic identified hotspot counties where DR-TB were clustered-targeted interventions might be important to reduce the burden of the disease in these hotspot counties. These analyses were conducted using tools provided in ArcGIS. ${ }^{12}$

In conducting a multivariable Bayesian spatial analysis, we assumed that the number of people with DR-TB at the county level followed a Poisson distribution. An initial bivariate analysis was conducted and variables with $\mathrm{p}<0.2$ were selected for the multivariable Bayesian spatial model. We constructed three different models using WinBUGS V.1.4.3 software (Medical Research Council Biostatistics Unit, Cambridge, UK). All variables selected from the bivariate analysis were incorporated as fixed effects in all three models. In the first model (model I), unstructured random effects were added. The assumptions were that: (1) the number of DR-TB cases in a county was independent of the number of DR-TB cases in other counties in the province, after accounting for the covariates and (2) the variance of random effects was homogeneous across the study area. To handle possible spatial dependency of cases and the violation of homogeneity of variance within each county due to the spatial nature of the data, a second model (model II) containing spatially structured random effects was constructed. These random effects used a Bayesian smoothing conditional autoregressive (CAR) structure ${ }^{13}$ Finally, a third model (model III) containing the covariates and both the unstructured and spatially structured random effects, was constructed as follows:

$$
\mathrm{Yi} \sim \operatorname{Poisson}(\mu \mathrm{i})
$$




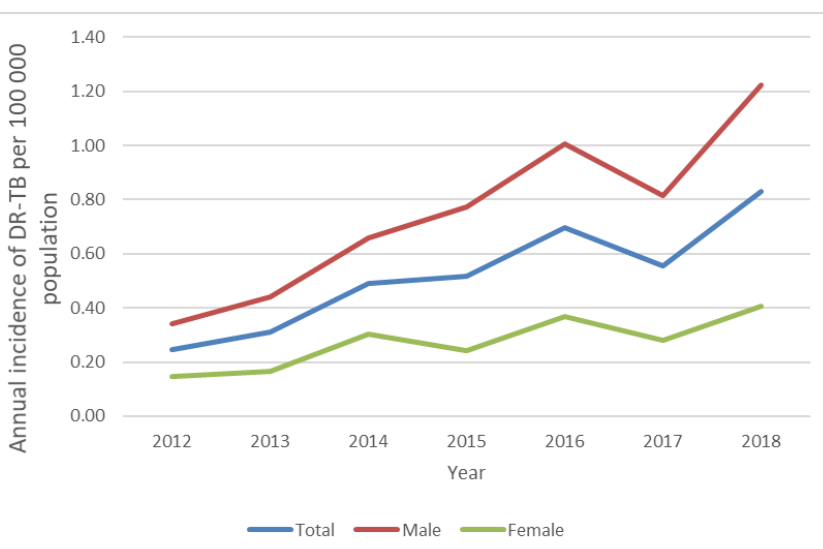

Figure 1 Annual incidence of notified drug-resistant tuberculosis (DR-TB) per 100000 population in Hunan Province, China, from 2012 to 2018.

where $Y$, the observed number of DR-TB cases in county $i$, was assumed to follow a Poisson distribution with mean $\mu_{i}$; and the log of the mean was modelled as:

$$
\log \left(\mu_{\mathrm{i}}\right)=\log \left(\mathrm{E}_{\mathrm{i}}+\alpha+\sum_{\mathrm{k}=1}^{\mathrm{k}} \beta_{\mathrm{k}} \times \mathrm{X}_{\mathrm{k}}+\mathrm{U}_{\mathrm{i}}+\mathrm{V}_{\mathrm{i}}\right)
$$

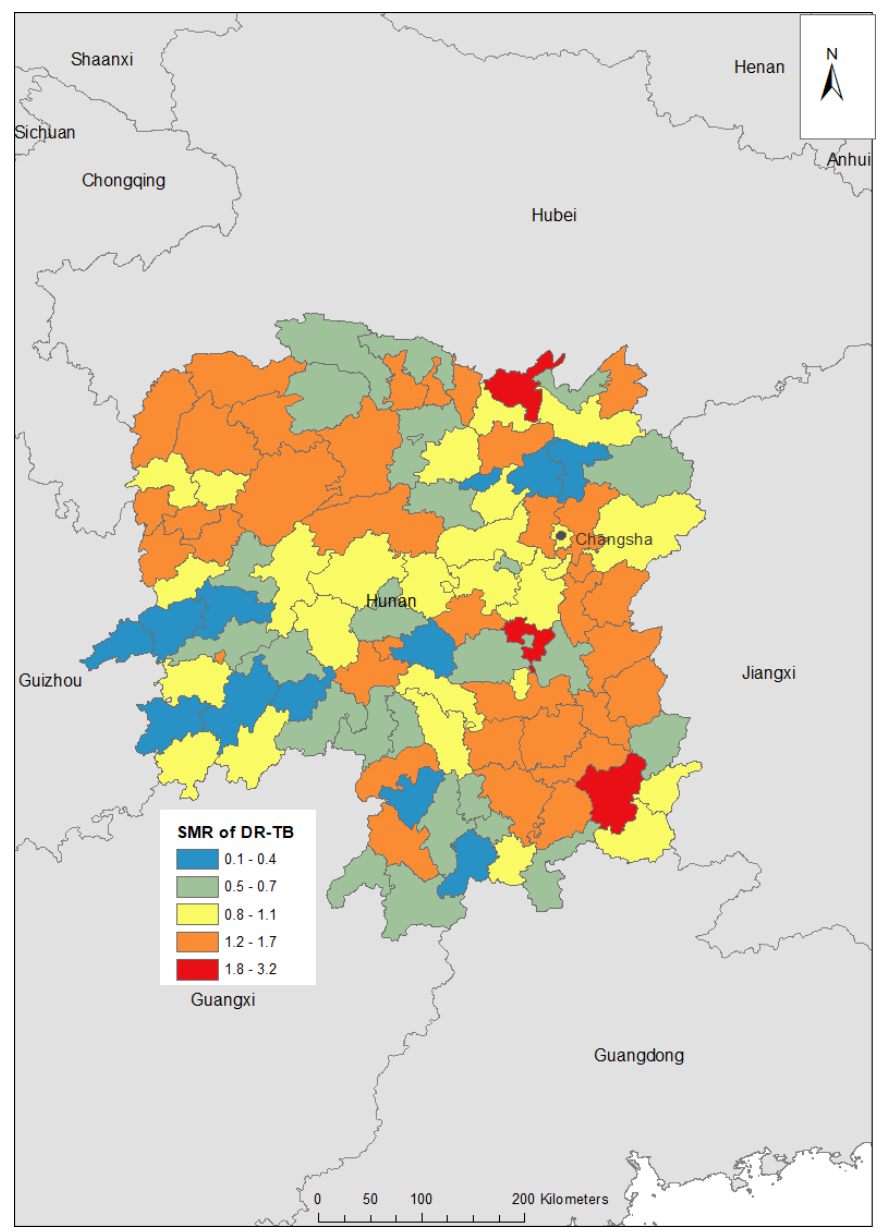

Figure 2 Spatial distribution of drug-resistant tuberculosis (DR-TB) standardised morbidity ratios (SMR) by County in Hunan Province, 2012-2018. where $E_{i}$ is the expected number of DR-TB cases in county $i, \alpha$ is the intercept, $\beta_{k}$ is the coefficient for covariate $X_{k}$ (where $K$ is the number of covariates), $U_{i}$ are the unstructured random effects and $V_{i}$ are the spatially structured random effects.

The spatially structured random effects $\left(\mathrm{V}_{\mathrm{i}}\right)$ were computed using a CAR structure by including an adjacency matrix to determine the spatial relationships between each pair of counties. The adjacency matrix for each county was generated using ArcGIS. A weight of 1 was given if two counties were neighbouring and a weight of 0 was given if two counties were not neighbouring. Two counties were considered to be neighbouring if they shared the same edges or corners (ie, queen contiguity). Prior probability distributions for the coefficients $(\beta)$, Ui and Vi were assumed to have normal distributions with a mean $=0$ and a precision (ie, inverse of variance $)=1 \times 10^{-6}$. For the intercept $(\alpha)$ a flat prior distribution was used (ie, a non-informative, improper prior with bounds $-\infty$ and $+\infty$ ). The prior for the precision of the unstructured and spatially structured random effects was assigned a non-informative gamma distribution with shape and scale parameters $=0.001$. The posterior distribution of the parameters was estimated from the prior and data likelihood information using a Bayesian Markov Chain Monte Carlo simulation approach with Gibbs sampling employed by WinBUGS. The models were run for 100000 iterations and convergence was successfully achieved after 80000 iterations for each of the models. Convergence of the model was checked by visual inspection of posterior kernel densities and history plots, and the posterior distribution of each parameter was stored for summary measures such as the posterior mean, SD and the $95 \%$ credible interval (CrI). The deviance information criterion (DIC) was also stored for model selection, where a lower DIC value was used to indicate the best-fitting, most parsimonious model. Choropleth maps of the raw data and model random effects were created using the ArcGIS software to show the spatial clustering of DR-TB in Hunan province before and after accounting for the covariates and to indicate hotspot areas for targeted interventions. Since the covariates have different units and scales of measurement, we standardised all the variables to a z-scale on the basis of their mean and SD but converted the relative risks back to the original scale for presentation of the results.

\section{Patient and public involvement}

No patients or public were involved in this research.

\section{RESULTS}

\section{Demographic characteristics of DR-TB}

A total of 323,340 TB cases were reported to Hunan TB Control Institute from 104 counties between 2013 and 2018. Off these, 2676 cases were DR-TB and included in the analysis. Twenty-seven DR-TB cases were from other provinces and were therefore excluded from the study. 


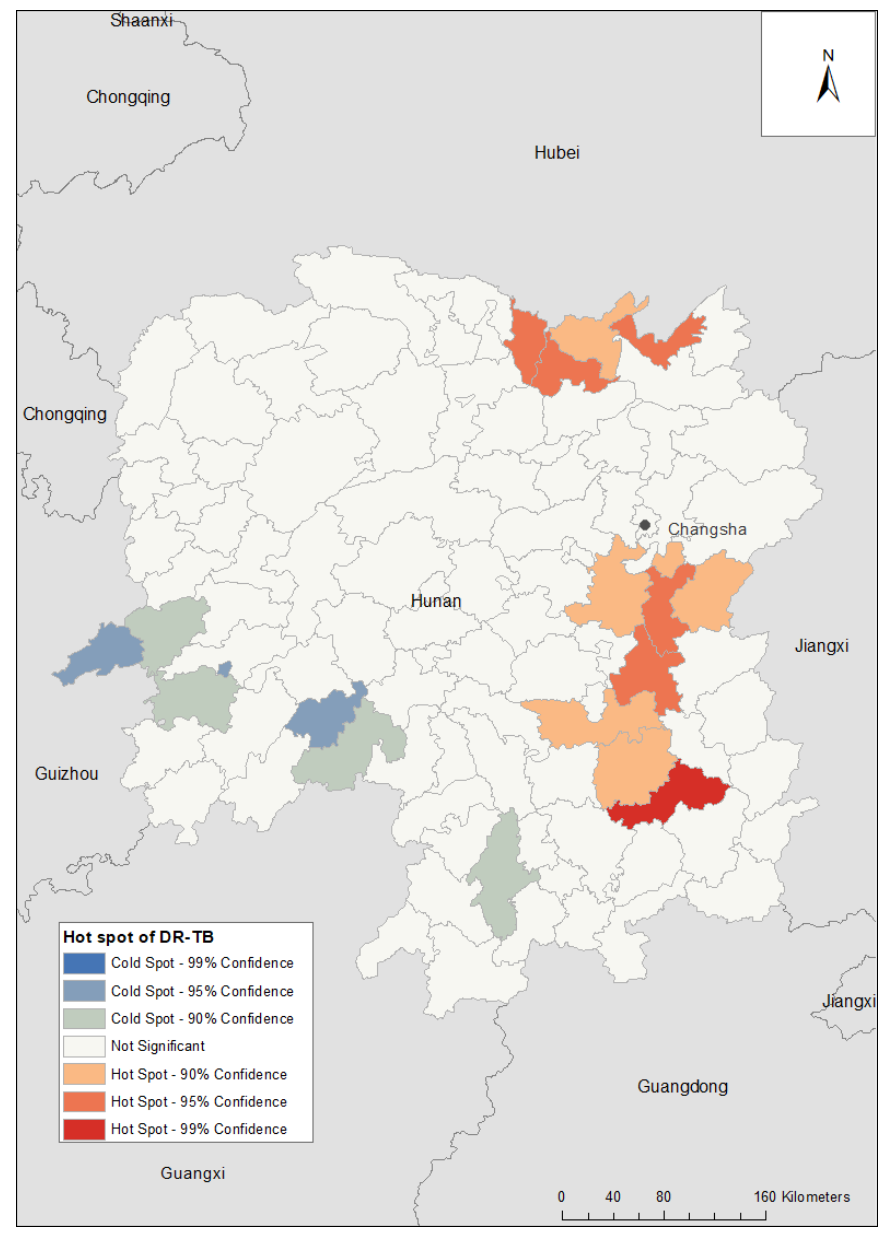

Figure 3 Spatial clustering of drug-resistant tuberculosis (DR-TB) incidence in Hunan province, 2012-2018, based on the Getis-Ord Giं ${ }^{*}$ statistic.

Of the 2649 included DR-TB patients, 1983 (74.8\%) were male and $1883(71.0 \%)$ were farmers by occupation. The mean age of patients was 46.5 years $(\mathrm{SD} \pm 15.16$, range 10-90 years). The largest number of cases was reported between September and December in every year, which accounted for $46.4 \%$ ( $n=1229)$ of the cases over the study period. Table 1 summarises the demographic and clinical characteristics of patients with DR-TB in Hunan province.

\section{Clinical characteristics of DR-TB}

The majority of DR-TB patients $(88.5 \%, \mathrm{n}=2345)$ had a history of first-line or second-line TB treatment and $11.4 \%$ $(n=304)$ were new cases of DR-TB. The number of new DR-TB cases increased from 15 cases in 2012 to 114 cases in 2018, and over two-thirds of all the cases $(37.5 \%)$ were reported in 2018. The proportion of XDR-TB among all DR-TB was $3.3 \%(n=89)(95 \%$ CI $2.7 \%$ to $4.1 \%)$ overall, and increased from $2.8 \%$ in 2012 to $4.4 \%$ in 2018 . The mean age of patients with XDR-TB was higher at 49.5 years ( $\mathrm{SD} \pm 15.4$ years) than the mean age of patients with MDR-TB at 46.2 years $(S D+15.1$ years $)(p=0.05)$. Half $(50.0 \% ; \mathrm{n}=1337)$ of the patients were taking treatment at the time of this study; treatment outcomes were not registered for 25 patients $(0.95 \%)$. Of 1287 DR-TB patients with registered treatment outcomes, $434(33.8 \%)$ were cured, $198(15.3 \%)$ completed treatment, $92(7.1 \%)$ died, $108(8.3 \%)$ had treatment failure and 455 (35.3\%) were lost to follow-up. Half $(50.9 \% \mathrm{n}=655)$ of the patients had poor treatment outcomes.

\section{Incidence rate of notified DR-TB}

The 6-year incidence rate of notified DR-TB in Hunan province was 3.9 per 100000 population. The overall drug-resistant testing coverage in Hunan Province has increased from $0.4 \%$ in 2013 to $1.3 \%$ in 2018 (online supplemental figure S1). Similarly, the annual incidence rate of notified DR-TB increased significantly over time from 0.25 per 100000 population in 2012 to 0.83 per 100 000 population in $2018(\mathrm{p}=0.002)$ (figure 1). The highest SMR) and incidence of notified DR-TB over the 6-year period were reported in Huarong county (12.6 per 100 000 population) and Hengshan county (11.0 per 100000 population) (figure 2). The incidence rate of notified DR-TB at prefecture level is presented in online supplemental figure S2.

\section{Spatial clustering of DR-TB}

Substantial spatial heterogeneity in DR-TB was observed, based on our clustering analyses. Using the Getis-Ord G statistic, 12 counties were identified as hotspots for DR-TB in Hunan province and 8 counties were cold spots (figure 3). The hotspot counties were in the north and east parts of Hunan province, while the cold spot counties were located in the south and west of the province. Anselin Local Moran's I statistic showed high-high and low-low clustering of DR-TB at county-level in Hunan province (online supplemental figure S3). Important changes in the spatial clustering of notified DR-TB was observed overtime in Hunan province (online supplemental figure S4).

\section{Ecological-level factors associated with spatial clustering of DR-TB}

Based on the lower DIC value identified from the multivariate Bayesian regression models, the model which contained the spatially structured random effect only (model II) was the best fit. The best-fitting model indicated that the incidence rate of notified DR-TB was significantly associated with urban communities (ie, percentage of urban residence in the counties (\%)) (RR: 1.02; 95\% CrI: 1.01 to 1.20$)$. Several ecological level factors such as sex, residence, gross domestic product and the mortality rate as well as healthcare access variables such as the number of health institutions (ie, hospitals and clinics) and number of medical personnel, and climatic variables such as monthly average temperature, annual precipitation and annual mean sunshine hours in a county were not significantly associated with the incidence rate of notified DR-TB in Hunan province (table 2). After accounting for the ecological-level factors in the model, the posterior mean of spatially structured random effects was found to be clustered in the province (figure 4). This indicates 
Table 2 A multivariate Bayesian poisson regression model with spatially structured and spatially unstructured random effects for DR-TB in Hunan province, China, 2012-2018

\begin{tabular}{|c|c|c|c|}
\hline & $\begin{array}{l}\text { Spatially unstructured } \\
\text { model }\end{array}$ & $\begin{array}{l}\text { Spatially structured } \\
\text { model }\end{array}$ & $\begin{array}{l}\text { Both spatially } \\
\text { structured and } \\
\text { unstructured model }\end{array}$ \\
\hline Variables & (RR (95\%Crl) & (RR (95\%Crl) & (RR (95\%Crl) \\
\hline \multicolumn{4}{|l|}{ Socioeconomic and demographic factors } \\
\hline Proportion of male in a county & 0.96 (0.87 to 1.07$)$ & 0.93 (0.82 to 1.07$)$ & 0.96 (0.86 to 1.08$)$ \\
\hline Percentage of urban residence in the counties & 1.09 (1.08 to 1.30$)$ & $1.02(1.01$ to 1.20$)$ & $1.04(1.02$ to 1.25$)$ \\
\hline Annual birth rate in the county (\%) & 0.97 (0.85 to 1.12$)$ & $1.00(0.85$ to 1.17$)$ & $0.98(0.85$ to 1.15$)$ \\
\hline Annual death rate in the county (\%) & $1.01(0.84$ to 1.20$)$ & $1.04(0.88$ to 1.23$)$ & 1.02 (0.85 to 1.22$)$ \\
\hline \multicolumn{4}{|l|}{ Healthcare access } \\
\hline Contraceptive use rate of the county (\%) & $0.93(0.82$ to 1.05$)$ & 0.97 (0.84 to 1.11$)$ & 0.94 (0.83 to 1.08$)$ \\
\hline Monthly average temperature $\left({ }^{\circ} \mathrm{C}\right)$ & $1.13(1.00$ to 1.28$)$ & 1.00 (0.85 to 1.18$)$ & $1.08(0.92$ to 1.24$)$ \\
\hline Annual total precipitation (mm) & 0.99 (0.85 to 1.14$)$ & $1.08(0.88$ to 1.33$)$ & 1.01 (0.85 to 1.23$)$ \\
\hline Monthly sunshine hours ( $\mathrm{kJ} / \mathrm{m}^{2} /$ day) & 1.15 (1.00 to 1.32$)$ & $1.02(0.80$ to 1.26$)$ & 1.11 (0.90 to 1.32$)$ \\
\hline \multicolumn{4}{|l|}{ Heterogenicity } \\
\hline Variance of spatially unstructured random effect $(\sigma 2)$ & 4.56 (3.05 to 6.73$)$ & & 6.21 (3.47 to 72.77 ) \\
\hline Variance of spatial structured random effect $(\sigma 2)$ & & $1.10(0.73$ to 1.64$)$ & $6.39(1.11$ to 729.60$)$ \\
\hline Intercept (alpha) & $-0.14(-0.25$ to -0.03$)$ & $-0.12(-0.19$ to -0.06$)$ & $-0.13(-0.23$ to -0.04$)$ \\
\hline DIC & 689.9 & 498.1 & 603.2 \\
\hline
\end{tabular}

*GDP ( $¥ 10000$ per person per year; $¥ 1=U S \$ 0.15)$.

$\mathrm{Crl}$, credible interval; DIC, deviance information criterion; GDP, gross domestic product.

that a substantial amount of county level heterogeneity in DR-TB remained unexplained by the ecological level factors included in our models. Geographical distribution of healthcare access variables such as number of health institutions (online supplemental figure S5), number of beds (online supplemental figure S6) and number of medical personnel (online supplemental figure S7) are presented in online supplemental file 1.

\section{DISCUSSION}

Our study detected spatial clustering in the incidence of notified DR-TB in Hunan Province, China. Counties located in the north and west part of Hunan province had high incidence of notified DR-TB. Previous studies conducted in South Africa, ${ }^{14-17}$ Moldova $^{18}$ and Ethiopia ${ }^{19}$ as well as studies conducted in other parts of China ${ }^{20} 21$ have also showed spatial clustering of DR-TB at the subnational level. The substantial heterogenicity in DR-TB indicates an imperative for more regular surveillance to detect emerging and existing hotspots for targeted interventions to prevent DR-TB, especially in settings where there are limited resources. Understanding the spatial distribution of DR-TB and identifying areas with the highest incidence of notified DR-TB is a first step towards reducing the burden of DR-TB. ${ }^{22}$

Our study also showed that the annual incidence of notified DR-TB in Hunan province steadily increased over time, from 0.25 per 100000 population in 2012 to 0.83 per 100000 population in 2018. There could be two reasons for this finding. The first reason could be due to a real increase in numbers of patients with DR-TB in the province as a result of local transmission of drug-resistant strains (ie, primary DR-TB) or due to the development of drug-resistant strains during a course of first-line TB treatment (ie, acquired or secondary DR-TB). Since the number of patients who were lost to follow-up in Hunan province was high at $35 \%$ in this study and $27 \%$ in a previous study, ${ }^{9}$ the risk of primary transmission of DR-TB in the community as well as the development of acquired DR-TB is high. Whole genome sequencing would be important to investigate this and to identify the main sources of DR-TB transmission in the province. The second reason could be due to improved diagnostic services and the introduction of rapid molecular diagnostic methods such as line probe assays and Xpert MTB/RIF. The impact of new molecular diagnostic test 


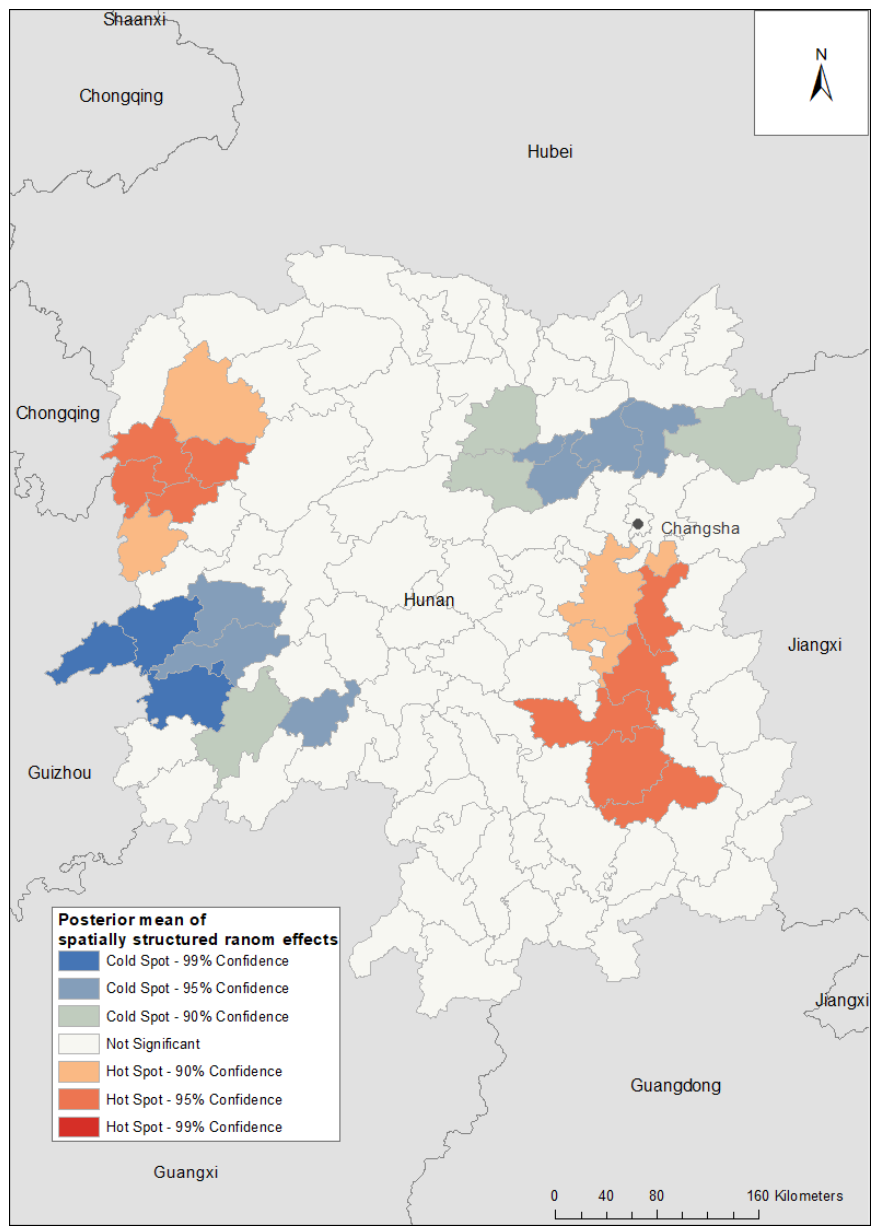

Figure 4 Posterior mean of spatially structured random effects for drug-resistant tuberculosis showing probability of being a hotspot $(R r>1)$ or cold spot $(R r<1)$ in Hunan province, 2012-2018.

on DR-TB incidence and reasons for increasing DR-TB in the province require further investigation.

This study also found that the incidence of notified DR-TB was significantly associated with residence in urban counties. Similarly, previous studies conducted in Ethiopia have reported that urban residence is associated with TB and DR-TB incidence. ${ }^{19}{ }^{23}$ However, unlike previous studies, several socioeconomic, demographic and climatic factors were not associated with clustering of DR-TB in Hunan province, and after accounting for these variables in the model, the spatial clustering of DR-TB remained. Our study included only one province, where ecological level variables such as climatic and economic factors could be relatively homogeneous and may not explain the local variation of the disease. Additionally, our model did not include other important covariates such as levels of HIV infection, diabetes, poverty and smoking that might better explain spatial variation, as these variables were not available in our dataset. A larger nationwide study might be required to fully understand the impacts of ecological-level factors on the spatial clustering of DR-TB in China.
Using routinely collected surveillance data from the provincial TB Programme, we produced maps showing the counties where DR-TB were clustered. These may help Provincial authorities to design strategies and target interventions for the prevention and control of TB and DR-TB. However, the study used secondary data available from the Hunan TB Control Institute, which may not reveal the true burden of the disease in the community because in some counties with high DR-TB, only those cases that are at highest risk of having DR-TB may receive a DST. Future studies should incorporate national TB and DR-TB survey, as well as whole genome sequencing data to better identify geographical areas at increased risk of DR-TB.

\section{Conclusion}

In this study, we found that the annual incidence rate of notified DR-TB increased over time and spatial clustering of DR-TB was detected in the North and East parts of Hunan province. This finding suggested that targeting interventions to highest risk areas and population groups would help to reduce the burden and to prevent the ongoing transmission of DR-TB.

\section{Author affiliations}

${ }^{1}$ Faculty of Health Sciences, Curtin University, Bentley, Western Australia, Australia

${ }^{2}$ Telethon Kids Institute, Nedlands, Western Australia, Australia

${ }^{3}$ Department of Tuberculosis Control, Tuberculosis Control Institute of Hunan

Province, Changsha, Hunan, China

${ }^{4}$ Department of Director's Office, Hunan Tuberculosis Control Institute, Changsha, Hunan, China

${ }^{5}$ Department of MDR-TB, Internal Medicine, Hunan Chest Hospital, Changsha, China ${ }^{6}$ Research School of Population Health, Australian National University, Canberra, ACT, Australia

${ }^{7}$ Public Health Sciences, Karolinska Institute, Stockholm, Sweden

${ }^{8}$ School of Public Health, The University of Sydney, Sydney, New South Wales, Australia

Acknowledgements The authors would like to thank those who were participating in the data translation from Mandarin to English.

Contributors KAA, KV, DG, ACAC and ZX conceived and designed the study. KAA, $H Y, L B, Y T$ and $Z X$ supervised the data translation process. KAA and ZX prepared the draft manuscript and KV improved the drafted manuscript. KV and ACAC advised the overall data analysis and the development of the manuscript. All the authors involved with critical revision of the manuscript and approved the final version.

Funding This study was funded by the China in the World Grant (CIW) 2019 support. Grant number is not applicable.

Disclaimer The contents are the responsibility of the authors and do not necessarily reflect the views of the funder. The funder had no role in study design, data collection and analysis, interpretation of data, decision to publish, or in writing the manuscript.

Competing interests None declared.

Patient consent for publication Not required.

Ethics approval Ethical clearance was obtained from the Australian National University (HRE11087) and Curtin University Human Research Ethics Committees (HRE2019-0581). Additional approval for the study and permission to access the DR-TB data was obtained from the Hunan Tuberculosis Control Institute. Since we used data aggregated at county level. All methods were performed in accordance with the approved protocol by the ethics committee.

Provenance and peer review Not commissioned; externally peer reviewed. 
Data availability statement Data are available on reasonable request. The data that support the findings of this study are available from the corresponding author on reasonable request.

Supplemental material This content has been supplied by the author(s). It has not been vetted by BMJ Publishing Group Limited (BMJ) and may not have been peer-reviewed. Any opinions or recommendations discussed are solely those of the author(s) and are not endorsed by BMJ. BMJ disclaims all liability and responsibility arising from any reliance placed on the content. Where the content includes any translated material, BMJ does not warrant the accuracy and reliability of the translations (including but not limited to local regulations, clinical guidelines, terminology, drug names and drug dosages), and is not responsible for any error and/or omissions arising from translation and adaptation or otherwise.

Open access This is an open access article distributed in accordance with the Creative Commons Attribution Non Commercial (CC BY-NC 4.0) license, which permits others to distribute, remix, adapt, build upon this work non-commercially, and license their derivative works on different terms, provided the original work is properly cited, appropriate credit is given, any changes made indicated, and the use is non-commercial. See: http://creativecommons.org/licenses/by-nc/4.0/.

\section{ORCID iD}

Kefyalew Addis Alene http://orcid.org/0000-0002-1904-4682

\section{REFERENCES}

1 WHO. Global tuberculosis report 2019. Geneva: World Health Organization, 2019.

2 Paulson T. A mortal foe. Nature 2013;502:S1-2.

3 Assembly G. Sustainable development goals. SDGs), transforming our world: the. 2015, 2030.

4 Uplekar M, Weil D, Lonnroth K, et al. WHO's new End TB Strategy. The Lancet 2015;385:1799-801.

5 Zhang J, Gou H, Hu X, et al. Status of drug-resistant tuberculosis in China: a systematic review and meta-analysis. Am J Infect Control 2016;44:671-6.

6 Zhao Y, Xu S, Wang L, et al. National survey of drug-resistant tuberculosis in China. New England Journal of Medicine 2012;366:2161-70.

7 Zhao L-li, Chen Y, Chen Z-nan, et al. Prevalence and molecular characteristics of drug-resistant Mycobacterium tuberculosis in Hunan, China. Antimicrob Agents Chemother 2014;58:3475-80.

8 Ding P, Li X, Jia Z, et al. Multidrug-Resistant tuberculosis (MDR-TB) disease burden in China: a systematic review and spatio-temporal analysis. BMC Infect Dis 2017;17:57.
9 Alene KA, Yi H, Viney K, et al. Treatment outcomes of patients with multidrug-resistant and extensively drug resistant tuberculosis in Hunan Province, China. BMC Infect Dis 2017;17:573.

10 Alene KA, Viney K, Yi H, et al. Comparison of the validity of smear and culture conversion as a prognostic marker of treatment outcome in patients with multidrug-resistant tuberculosis. PLoS One 2018;13:e0197880.

11 Ge E, Li D, Luo M, et al. Transmission of multidrug-resistant tuberculosis in Shanghai: roles of residential status. Int J Tuberc Lung Dis 2018;22:1462-8.

12 ArcGIS Desktop: Release 10. 6.1 [program]. USA 2015.

13 Besag J, York J, Molli A. Bayesian image restoration, with two applications in spatial statistics. Ann Inst Stat Math 1991;43:1-20.

14 Nelson KN, Shah NS, Mathema B, et al. Spatial patterns of extensively drug-resistant tuberculosis transmission in KwaZuluNatal, South Africa. J Infect Dis 2018;218:1964-73.

15 Mclntosh Al, Jenkins HE, White LF, et al. Using routinely collected laboratory data to identify high rifampicin-resistant tuberculosis burden communities in the Western Cape Province, South Africa: a retrospective spatiotemporal analysis. PLoS Med 2018;15:e1002638.

16 Smith CM, Lessells R, Grant AD, et al. Spatial clustering of drugresistant tuberculosis in Hlabisa subdistrict, KwaZulu-Natal, 20112015. Int J Tuberc Lung Dis 2018;22:287-93.

17 Peterson ML, Gandhi NR, Clennon J, et al. Extensively drug-resistant tuberculosis 'hotspots' and sociodemographic associations in Durban, South Africa. Int J Tuberc Lung Dis 2019;23:720-7.

18 Jenkins HE, Plesca V, Ciobanu A, et al. Assessing spatial heterogeneity of multidrug-resistant tuberculosis in a high-burden country. European Respiratory Journal 2013;42:1291-301.

19 Alene KA, Viney K, McBryde ES, et al. Spatial patterns of multidrug resistant tuberculosis and relationships to socio-economic, demographic and household factors in Northwest Ethiopia. PLoS One 2017;12:e0171800.

20 Jiang Q, Liu Q, Ji L. Citywide Transmission of Multidrug-resistant Tuberculosis Under China's Rapid Urbanization: A Retrospective Population-based Genomic Spatial Epidemiological Study. Clinical Infectious Diseases 2019.

21 Chen W, Liu Z, Wang X, et al. Spatial analysis of patients with multi-drug resistant pulmonary tuberculosis between 2009 and 2012 in eastern China. International Journal of Infectious Diseases 2016;45:221.

22 Alene KA, Adane AA, Yifiru S, et al. Knowledge and practice of health workers about control and prevention of multidrug-resistant tuberculosis in referral hospitals, Ethiopia: a cross-sectional study. BMJ Open 2019;9:e022948.

23 Alene KA, Viney K, McBryde ES, et al. Spatiotemporal transmission and socio-climatic factors related to paediatric tuberculosis in northwestern Ethiopia. Geospat Health 2017;12:575. 